\title{
Hierarchical Sizing and Power Distribution Strategy for Hybrid Energy Storage System
}

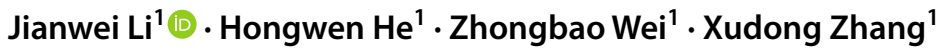

Received: 2 January 2021 / Accepted: 6 September 2021 / Published online: 28 October 2021

(c) The Author(s) 2021

\begin{abstract}
This paper proposes a hierarchical sizing method and a power distribution strategy of a hybrid energy storage system for plug-in hybrid electric vehicles (PHEVs), aiming to reduce both the energy consumption and battery degradation cost. As the optimal size matching is significant to multi-energy systems like PHEV with both battery and supercapacitor (SC), this hybrid system is adopted herein. First, the hierarchical optimization is conducted, when the optimal power of the internal combustion engine is calculated based on dynamic programming, and a wavelet transformer is introduced to distribute the power between the battery and the SC. Then, the fuel economy and battery degradation are evaluated to return feedback value to each sizing point within the hybrid energy storage system sizing space, obtaining the optimal sizes for the battery and the SC by comparing all the values in the whole sizing space. Finally, an all-hardware test platform is established with a fully active power conversion topology, on which the real-time control capability of the wavelet transformer method and the size matching between the battery and the SC are verified in both short and long time spans.
\end{abstract}

Keywords Plug-in hybrid electric vehicles $\cdot$ Hybrid energy storage $\cdot$ Battery energy storage $\cdot$ Hierarchical sizing

$\begin{array}{ll}\text { Abbreviations } \\ \text { CTUDC } & \text { Chinese typical urban driving cycle } \\ \text { DC } & \text { Direct current } \\ \text { DM } & \text { Driving motor } \\ \text { DP } & \text { Dynamic planning } \\ \text { EMS } & \text { Energy management system } \\ \text { EV } & \text { Electric vehicle } \\ \text { HESS } & \text { Hybrid energy storage system } \\ \text { HEV } & \text { Hybrid electric vehicle } \\ \text { HIL } & \text { Hardware-in-the-loop } \\ \text { ICE } & \text { Internal combustion engine } \\ \text { PHEV } & \text { Plug-in hybrid electric vehicle } \\ \text { RFCC } & \text { Rain-flow cycle counting } \\ \text { SC } & \text { Supercapacitor } \\ \text { WT } & \text { Wavelet transformer }\end{array}$

Hongwen $\mathrm{He}$

hwhebit@bit.edu.cn

1 National Engineering Laboratory for Electric Vehicles, School of Mechanical Engineering, Beijing Institute of Technology (BIT), Beijing, China

\section{Introduction}

The electrification of the automobile industry is a critical step toward addressing the energy dilemma and protecting the environment $[1,2]$. The adoption of plug-in hybrid electric vehicles (PHEVs) makes it possible to reduce the consumption of fossil fuels and provides a more positive environmental impact [3]. Some countries have devised both short- and long-term policies to promote the use of PHEVs [4]. The study of the hybrid energy storage system has shifted its focus to how to accurately estimate the internal state of the system, delay battery life degradation, and realize coordinated and optimized control of power and energy [5, 6]. A significant number of studies have investigated PHEV management to reduce fuel consumption and extend battery life to increase the economic performance of PHEVs [7, 8].

Many scholars have developed different strategies to boost the economics of PHEVs [9]. Reference [10] proposed a back-to-back competitive learning mechanism-driven fuzzy logic-based energy storage approach to increase the fuel efficiency of a hybrid electric vehicle. The experimental results showed that the proposed method reduced the fuel consumption by $7 \%$ compared with the traditional energy management system (EMS). The reinforcement learning algorithm 
is used in Ref. [11] to reduce fuel consumption of the hybrid electric vehicle (HEVs), saving $2.04 \%$ more energy than stochastic dynamic programming-based energy management. To extensively investigate the impact of uncertainty on electric vehicle (EV) charging efficiency, probabilistic modeling of $\mathrm{EV}$ charging patterns in residential delivery networks was performed [12]. However, certain operating modes or control algorithms increase fuel economy by overloading the battery grid, which reduces battery lifespan [13]. Thus, it is necessary to constrain battery degradation cost for EMS of PHEV [14]. In typical PHEVs, there is a mutual influence between the use of energy and battery degradation, i.e., reduced energy consumption implies a higher efficiency of the battery, which would eventually reduce battery life and increase its degradation cost [15].

Over years, the hybrid energy storage system has been developed with a strong prospect of enhancing the economic performance of PHEV, particularly power electronics and supercapacitor (SC) technology [8, 16, 17]. The lifespan of a $\mathrm{SC}$ is longer, as it has a much higher power density, allowing it to have an efficient energy output $[18,19]$. The rule-based energy management strategy is proposed in Ref. [20] for a battery/SC hybrid energy storage system to generate the battery current reference in a robust fractional-order slidingmode control, with hardware-in-the-loop (HIL) to test the efficacy of the proposed control scheme. In Ref. [6], the energy management technique generates the battery current reference, while the Lyapunov controller generates the current supercapacitor reference to ensure voltage stability of the direct current (DC) bus. The simulation and test proved the stable monitoring and smooth transfer during great load power [20].

Figure 1 illustrates an example of the hybrid energy storage system of the PHEV powertrain [21]. Similar to traditional vehicles, the engine provides propulsive torque directly to the wheels of HEV [22-24]. An electric motor driven by a battery pack is also mechanically attached to the driveline, which enables the engine to increase its power output. The torques produced by the motor are supplied to the wheels. The engine torque and motor torque

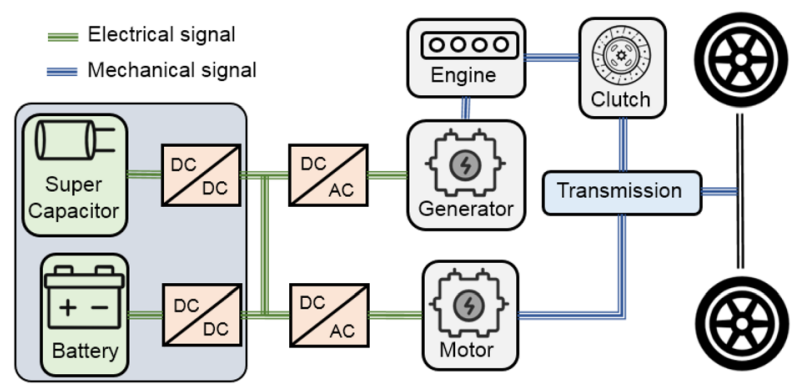

Fig. 1 Powertrain structure of PHEV hybrid storage system can be controlled separately, but each of the motors and engines has its speeds defined by the overall speed of the vehicle. A supercapacitor system is connected to the power bus of the PHEV energy system by a bi-directional DC-DC converter [8]. However, the distribution of power among three power sources with their different characteristics remains a challenge. Few papers considered battery degradation cost. If the operating points of the compressor, motor, and HESS are not optimized, the economic performance of PHEVs will suffer. To fill these research gaps, this paper proposes a new strategy to improve both fuel efficiency and battery longevity in PHEV energy management.

What is critical to the aforementioned studies is that the sizes of components in the multi-energy system should be established beforehand, and the following study on EMS performance was performed following the predetermined value. When the component size changes, the system performance is significantly affected. As a result, size matching is important in developing the best multi-energy system. Previous multi-energy system size-matching research has studied a variety of situations, including microgrids and transportation [15, 25]. Some researchers proposed global optimization methods [26-28]. Eldeeb et al. [29] developed a multi-objective optimization approach for minimizing the HESS system cost on a PHEV, in which weight and volume were taken into account while determining the best battery unit and SMES size. A hybrid method was proposed in the sizing process, which involves multi-objective optimization [30]. Furthermore, several scholars looked into statistical analysis. According to the statistical description of driving cycles, for example, Ravey et al. developed a technique for optimizing the size of HEV's power supply [31].

Therefore, the key contributions of this study are summarized as follows:

- A hierarchical sizing method that combines the dynamic energy management algorithm with the static sizing space is developed for PHEV.

- Under the hierarchical sizing framework, both the battery degradation and fuel economy are evaluated quantitatively.

- An all-hardware HESS platform is designed and implemented to verify the real-time control capability of the wavelet transformer (WT) and to prove the effectiveness of the size matching between the battery and SC.

The rest of the paper is organized as follows: Sect. 2 gives a detailed introduction about the main methods developed and applied, including the hierarchical sizing method, optimal size-searching method, rain-flow cycle counting algorithm, WT method, and the hardware design. The test 
platform and all the real-time experiential results are presented in Sect. 3. Conclusions are provided in Sect. 4.

\section{Hierarchical Sizing Study}

The concept of the hierarchical model has been widely used in the industrial applications, and the idea of hierarchical sizing is significant for multi-energy systems [32]. The hierarchical sizing study is conducted based on both the dynamic planning (DP) and WT, focusing on the overall HESS capacity and each power component. The structure and workflow of the proposed hierarchical sizing study are shown in Fig. 2, which is divided into three levels.

(1) At the first level, the power requirement of a PHEV is split into two parts: the power requirement from the internal combustion engine (ICE) and that from the driving motor (DM). The DP algorithm is used to achieve the optimum power distribution between the ICE and DM based on the Chinese typical urban driving cycle (CTUDC) and the performance maps of ICE and DM. Then, the power and energy requirement for the hybrid energy storage can be obtained.

(2) At the second level, the wavelet transform algorithm is used to share the power inside the HESS between the $\mathrm{SC}$ and the battery. The working principle of the WT is that the battery should be kept from the instantaneous high power requirement, which means the WT needs to filter both the high-frequency and high power component.

(3) At the third level, a comprehensive sizing study is developed, searching the SC-battery sizing space to both the battery degradation and fuel cost. The battery life-cycle costs are quantified using the rain-flow cycle counting (RFCC) algorithm. A detailed introduction about the comprehensive sizing study for the battery

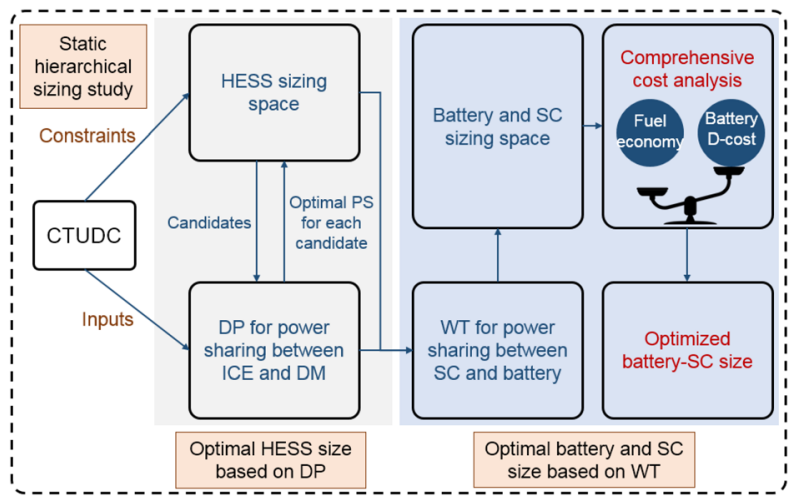

Fig. 2 Hierarchical sizing study and the SC and the RFCC will be introduced in Sects. 2.2 and 2.3 , respectively.

DP is based on the optimal control theory and can always generate the most fuel-efficient results while dealing with the PHEV energy management problem. DP can solve the powertrain control problem for most of the HEV/ PHEV structures developed currently. Actually, it is not a feasible approach for real-time application given the computational cost and the requirement of priori knowledge of the power profile (unrealistic for practical applications). For the real-time application, the DP could be used to deal with time-window optimization with a very short time span, such as several seconds. Therefore, globally optimal control is normally implemented offline and serves as a benchmark to explore the fuel economy potential. DP is used as a recalibration method to improve and evaluate the performance of the presented energy management. For example, DP is employed to locate the optimized control actions at each stage by minimizing the fuel consumption cost over a certain driving cycle. Therefore, the key control parameters under DP control could be extracted to improve the proposed energy strategy. The DP optimization results were used to recalibrate the proposed energy control strategy, and then an optimization-based control strategy could be acquired.

\subsection{Optimal Size-Searching Method}

The complemention of the battery and the SC lies in two levels: the first level for the sizing problem and the second level for the optimal energy management problem. For the sizing problem, there are four parameters, the nominal energy capabilities and power for both the battery and the SC, that need to be optimized for the HESS. For a given power demand, it is very difficult to conduct a comprehensive sizing study with a clear description of these four parameters and their relationships, as the parameters are coupled together. This paper uses a searching-cube-based framework to give a straightforward but comprehensive description of the parameters in the sizing study.

\subsection{Rain-Flow Cycle Counting}

The battery is generally considered to reach the end of life when more than $20 \%$ of its initial capacity has been used [33]. The RFCC algorithm is usually used for analyzing the fatigue of the metallic material. Many studies have already used the RFCC method to calculate the battery degradation cost, while, herein, the RFCC is used to obtain the irregular charging and discharging cycles. Basically, the cycle 


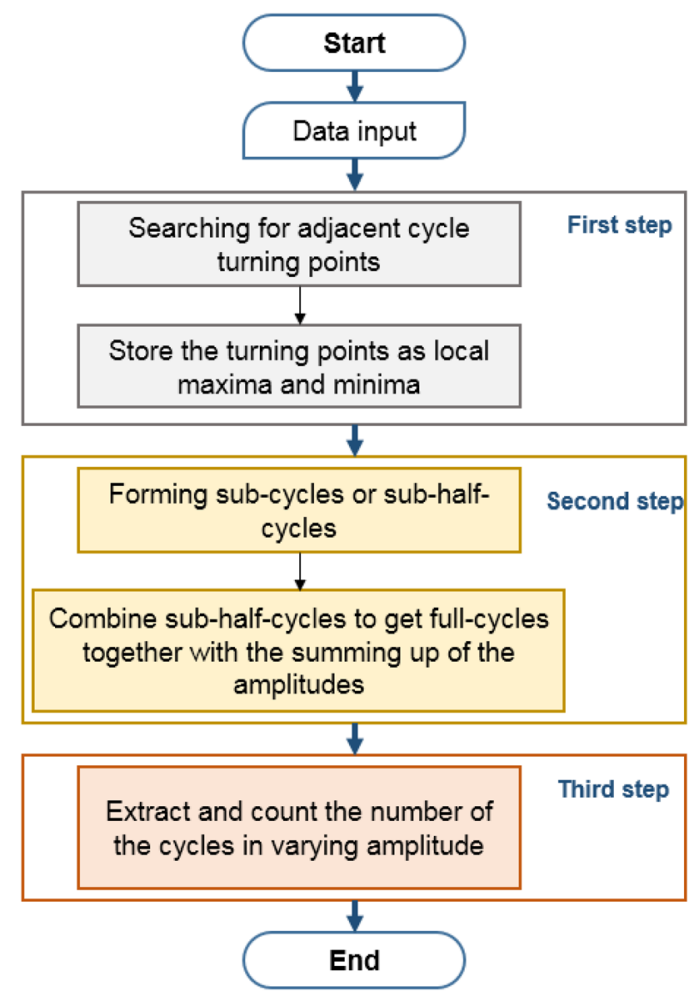

Fig. 3 RFCC method

counting can be achieved by the following three steps as shown in Fig. 3.

(1) Firstly, the data (for the battery, the data are the depth of discharge that presents the battery charge/discharge cycles) are pre-processed by searching for adjacent data points with the reverse polarity so that the local maxima and minima can be found and stored in a matrix.

(2) Secondly, compose full cycles by analyzing the turning points and combine these sub-cycles to get full cycles together with the summing up of the amplitudes.

(3) Thirdly, extract and count the number of cycles in varying amplitude store them for later use.

The detailed introduction of the RFCC method can be found in Ref. [34].

\subsection{Wavelet Transform for HESS Power Distribution}

The approximation and information of wavelet transform signals are at various frequency bands, using different resolution. The high-frequency components would cause significant and accelerated battery loss in PHEV, so the wavelet transform algorithm is used to separate the high-frequency components. The wavelet transforms the signal into several components with varying resolutions expressed as:

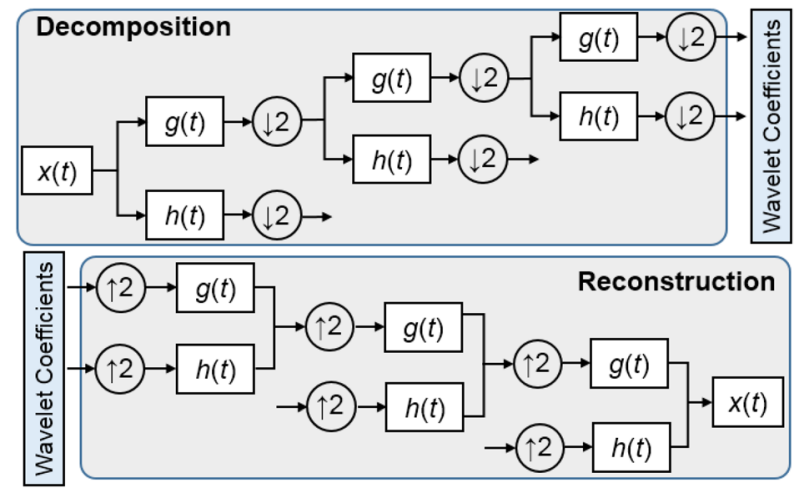

Fig. 4 Wavelet transform process

$x(t)=\sum_{k} a_{j, k} \phi_{j, k}(t)+\sum_{k} d_{j, k} \psi_{j, k}(t)+\sum_{k} d_{j-1, k} \psi_{j-1, k}(t)+\sum_{k} d_{1, k} \psi_{1, k}(t)$

where $a$ represents the smooth coefficient, $d$ represents the detail coefficient, $\phi$ is the father wavelet, and $\psi$ is the mother wavelet.

The discrete wavelet is defined as:

$W_{\psi}\left\{x(t)_{j, k}\right\}=2^{-j / 2} \sum_{j, k} x(t) \psi^{*}\left(2^{-j}-k\right)$

$\psi_{a, \tau}(t)=\frac{1}{\sqrt{|a|}} \psi\left(\frac{t-\tau}{a}\right)$

The 3-level wavelet transform is shown in Fig. 4 for highand low-frequency power processing. The $x(t)$ denotes the processed signal, which is decomposed into wavelet coefficients including detailed components and approximation components.

The power demands for the battery and supercapacitor are distributed using the wavelet transform. Figure 5 depicts the high-frequency and low-frequency components of the 3-level decomposition of $x(t)$. After the decomposition, the smooth and low-frequency component $f_{0}$ is obtained as the power demand for the battery. The remaining high-frequency components, $w_{2}, w_{1}$, and $w_{0}$, are assigned to the supercapacitor. The lithium-ion battery power requirement curve changes smoothly and slowly during wavelet decomposition, but at some point, there is still a high-frequency power demand in batteries. With the help of SCs, the high-frequency power requirements for the battery have been drastically reduced. Hence, battery life can be extended, which is a significant benefit of using the wavelet transform in HEV.

\subsection{Real-Time Control of HESS in PHEV}

Power balancing is the major challenge for the efficient use of energy storage devices in different applications, the 
achievement of which mainly depends on the control circuit. The real-time control scheme for HESS in PHEV is shown in Fig. 6.

The HESS control scheme is equipped with two DC/ DC converters. There are two inner current control loops ensured by the two DC/DC converters, which work together to maintain desirable output voltage from both the battery and the SC. Further, the appropriate power distribution between the two energy sources is achieved by generating proper current reference under the control algorithm. The WT is used to meet the power requirements,

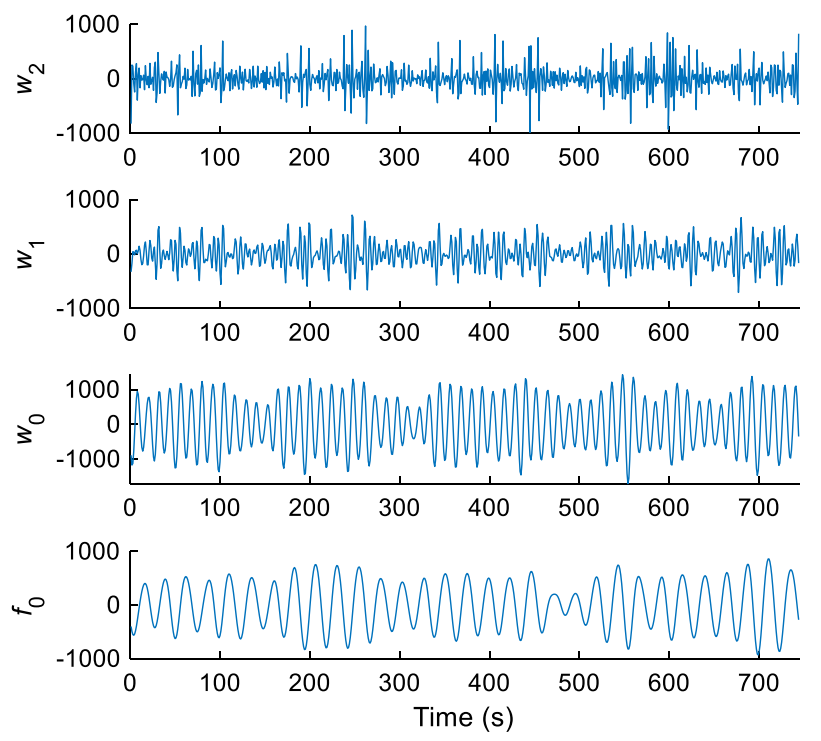

Fig. 5 3-level decomposition of electric power demands $\left(w_{0}, w_{1}, w_{2}\right.$, and $f_{0}$ components of the $\left.x(t)\right)$

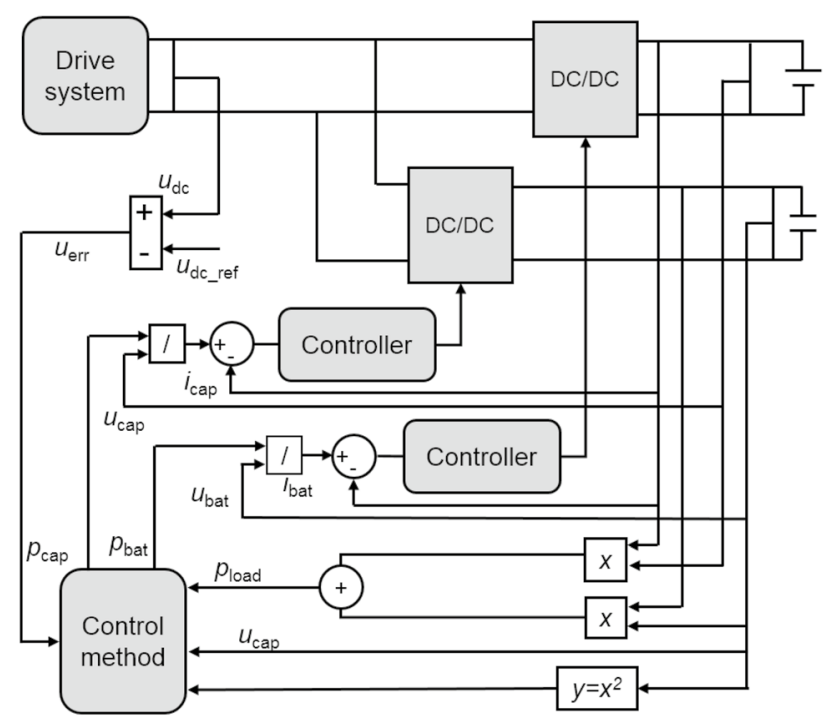

Fig. 6 Control scheme for HESS in PHEV by generating the references for the current control of the battery and the SC.

\section{Real-Time Tests and Results}

The CTUDC is applied for a city bus test. The standardized driving cycle is more than $5 \mathrm{~km}$ with a driving duration of more than $20 \mathrm{~min}$, and the typical profiles including the driving speed and acceleration in CTUDC are shown in Fig. 7.

\subsection{Hardware System Setup}

The dynamic performance of PHEV directly impacts the driving performance. The real-time performance of the proposed power distribution method is verified by hardware test, where the HESS test setup is shown in Fig. 8. It consists of energy storage devices (battery and SC), three bi-directional DC-DCs, a DC bus system, a DC filter, a controller, a digital storage oscilloscope, and a programmable load. The high-speed digital logic chip DSP28335 executes the proposed strategy and controls DC-DC converters.

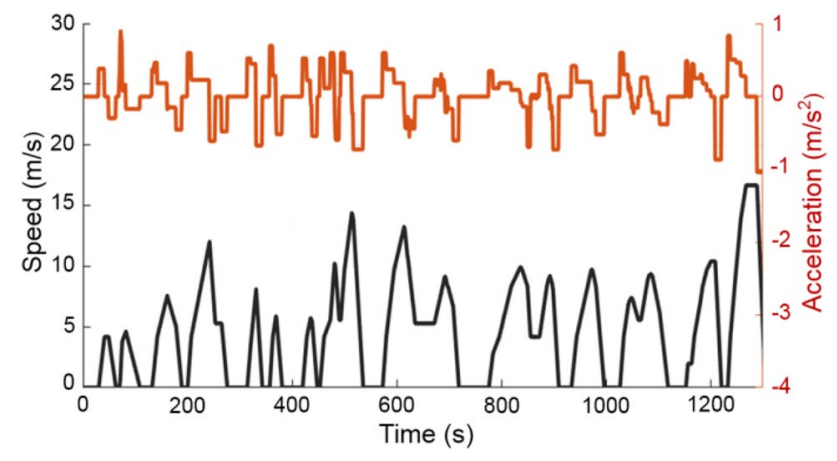

Fig. 7 Driving speed and acceleration in CTUDC

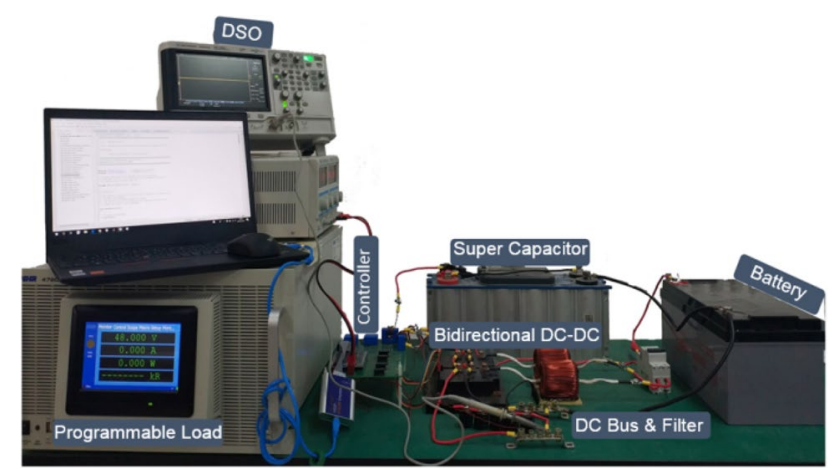

Fig. 8 Test setup of HESS 
The proposed matching capacity and the real-time control of the hybrid energy storage system is verified in two scenarios:

- Scenario 1: long-term CTUDC training of HESS in PHEV

- Scenario 2: comparison of the battery performances of the battery-only system and the hybrid system.

\subsection{Scenario 1: Long-Term CTUDC Training}

The proposed WT control method is used to distribute the power between the SC and the battery. Herein, one standard CTUDC cycle is used as the training case, and the results are shown in Fig. 9. As shown in Fig. 9b, the DM actual power is the targeted power requirement for the battery and the SC. As shown in Fig. 9a, the DM should work together with the ICE to meet the driving requirement. However, the optimal power contribution from the DM is determined by the DP. Therefore, the main control scheme is designed for the HESS rather than the whole PHEV system which includes the ICE. As shown by the orange line in Fig. 9b, the instantaneous high power component from the DM is transferred to the supercapacitor and the battery, and it is used to deal with the low-frequency component obtained after the WT. Figure 9c compares the battery current and SC current. The battery may still undergo some peak power, for example around the $500 \mathrm{~s}$ in the blue zone, when the SC deals with more power fluctuations
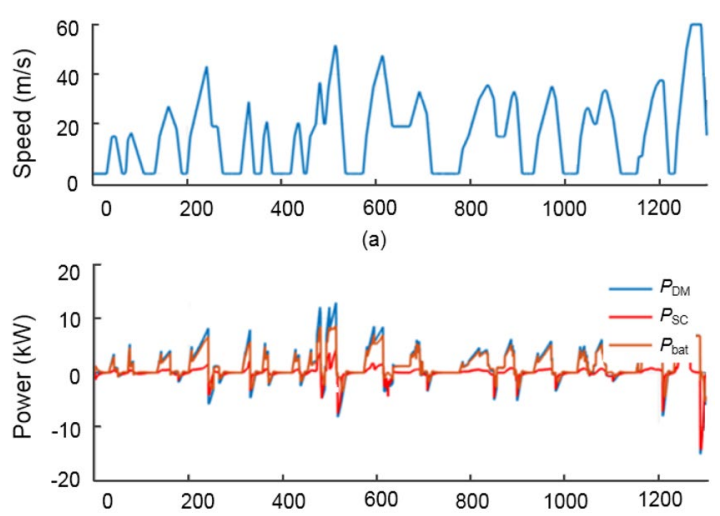

(b)

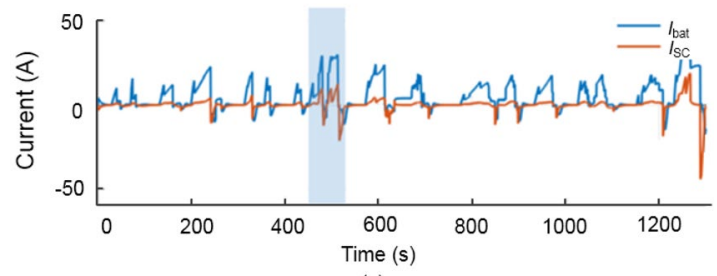

(c)

Fig. 9 Dynamic performance of HESS in PHEV at the same time. The test results show that the dynamic performance of the HESS conforms to the original design intention, which is that the battery mainly deals with the low-frequency power, whereas the SC takes care of the high-frequency fluctuations.

\subsection{Scenario 2: Comparison of Battery-Only System and HESS}

A case study is conducted with a rapid loading in the proposed platform, comparing the battery-only system and the hybrid system. The results are shown in Fig. 10.

As seen from Fig. 10b, the battery (the green curve) experiences a smooth change; while in Fig. 10a, it undergoes an instantaneous change. Further, as shown in the red zone of Fig. 10b, the DC bus voltage in HESS shows a smaller drop compared with that of the battery-only system. Thus, the SC operates more quickly than the battery, allowing it to provide a faster compensation to the DC bus voltage, demonstrating another advantage of the proposed hybrid system.

The bus voltage of the SC system and that of the battery system are approximately stable in the test, which indicates that the proposed EMS coordinates the operation of the SC and battery system reasonably, and the power requirements of DM are satisfied in real-time.

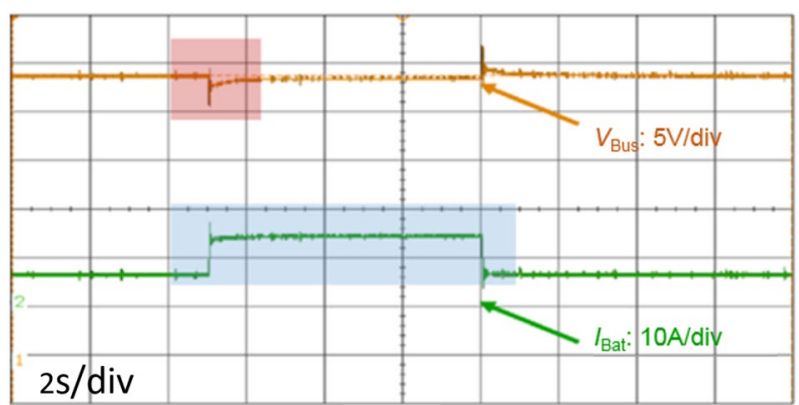

(a)

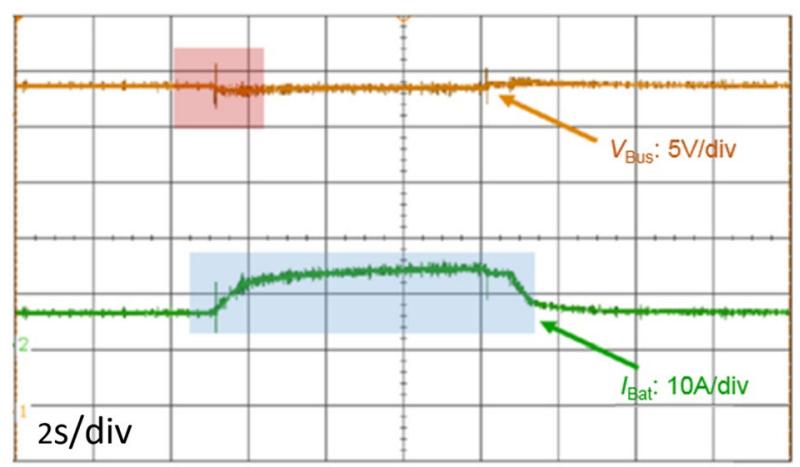

(b)

Fig. 10 Rapid loading test of (a) battery-only system and (b) hybrid system 


\section{Conclusions}

This paper proposes a hierarchical sizing approach and a hardware design for a hybrid energy storage device for PHEVs which helps to reduce energy consumption and the cost of battery degradation. Optimum energy is determined by dynamic programming on the internal combustion engine, then the power distribution between batteries, and $\mathrm{SC}$ is realized by a wavelet transformer. By scanning and comparing all values in the whole size space, the best sizes for the battery and the SC are obtained. Further, a power conversion topology is designed on an all-hardware platform. The tests validate the real-time control capacity as well as the size matching between the battery and the SC in both short and long time spans. Two scenarios are used to validate the capability and real-time control of the hybrid energy storage system. The highest battery c-rate limit is 8 , and the highest SC c-rate limit is 20 . The results prove that the bus voltage of the SC system and that of the battery system are stable, indicating that the proposed EMS coordinates the operation of the SC and the battery system in a reasonable manner.

Acknowledgements This work was supported by the Nature Science Foundation of China with Grant No. 51807008 and China Association for Science and Technology Youth Talent Promotion Project.

\section{Declarations}

Conflict of interest On behalf of all the authors, the corresponding author states that there is no conflict of interest.

Open Access This article is licensed under a Creative Commons Attribution 4.0 International License, which permits use, sharing, adaptation, distribution and reproduction in any medium or format, as long as you give appropriate credit to the original author(s) and the source, provide a link to the Creative Commons licence, and indicate if changes were made. The images or other third party material in this article are included in the article's Creative Commons licence, unless indicated otherwise in a credit line to the material. If material is not included in the article's Creative Commons licence and your intended use is not permitted by statutory regulation or exceeds the permitted use, you will need to obtain permission directly from the copyright holder. To view a copy of this licence, visit http://creativecommons.org/licenses/by/4.0/.

\section{References}

1. Xu, X., Dong, P., Liu, Y., et al.: Progress in automotive transmission technology. Automot. Innov. 1(3), 187-210 (2018)

2. Li, J., Zhang, M., Yang, Q., et al.: SMES/battery hybrid energy storage system for electric buses. IEEE Trans. Appl. Supercond. 26(4), 1-5 (2016)

3. Van Wieringen, M., Pop-Iliev, R.: Development of a dual-fuel power generation system for an extended range plug-in hybrid electric vehicle. IEEE Trans. Ind. Electron. 57(2), 641-648 (2009)
4. Neves, S.A., Marques, A.C., Fuinhas, J.A.: Technological progress and other factors behind the adoption of electric vehicles: empirical evidence for EU countries. Res. Transp. Econ. 74, 28-39 (2019)

5. Wang, Y., Wang, L., Li, M., et al.: A review of key issues for control and management in battery and ultra-capacitor hybrid energy storage systems. eTransportation. 100064 (2020)

6. Song, Z., Hou, J., Hofmann, H., et al.: Sliding-mode and Lyapunov function-based control for battery/supercapacitor hybrid energy storage system used in electric vehicles. Energy 122, 601-612 (2017)

7. Olaniyi, K.A., Ogunleye, A.A., Osifeko, T.M.: Review of strategies for hybrid energy storage management system in electric vehicle Application. J. Electr. Comput. Eng. 14(8), 224-232 (2020)

8. Wang, C., Xiong, R., He, H., et al.: Efficiency analysis of a bidirectional DC/DC converter in a hybrid energy storage system for plug-in hybrid electric vehicles. Appl. Energy 183, 612-622 (2016)

9. Gao, Y., Ehsani, M.: Design and control methodology of plug-in hybrid electric vehicles. IEEE Trans. Ind. Electron. 57(2), 633640 (2009)

10. Li, J., Zhou, Q., Williams, H., et al.: Back-to-back competitive learning mechanism for fuzzy logic based supervisory control system of hybrid electric vehicles. IEEE Trans. Ind. Electron. 67(10), 8900-8909 (2019)

11. Liu, T., Zou, Y., Liu, D., et al.: Reinforcement learning of adaptive energy management with transition probability for a hybrid electric tracked vehicle. IEEE Trans. Ind. Electron. 62(12), 7837-7846 (2015)

12. Ul-Haq, A., Cecati, C., El-Saadany, E.: Probabilistic modeling of electric vehicle charging pattern in a residential distribution network. Electr. Power Syst. Res. 157, 126-133 (2018)

13. Zhao, Z., Meng, Z., Li, L., et al.: Experimental study on the combustion and energy flows of vehicle engine under NEDC of cold start. Automot. Innov. 2(4), 314-327 (2019)

14. Hu, X., Jiang, J., Egardt, B., et al.: Advanced power-source integration in hybrid electric vehicles: multicriteria optimization approach. IEEE Trans. Ind. Electron. 62(12), 7847-7858 (2015)

15. Yang, Q., Li, J., Cao, W., et al.: An improved vehicle to the grid method with battery longevity management in a microgrid application. Energy 198, 117374 (2020)

16. Li, J., Wang, X., Zhang, Z., et al.: Analysis of a new design of the hybrid energy storage system used in the residential m-CHP systems. Appl. Energy 187, 169-179 (2017)

17. Camara, M.B., Gualous, H., Gustin, F., et al.: DC/DC converter design for supercapacitor and battery power management in hybrid vehicle applications-Polynomial control strategy. IEEE Trans. Ind. Electron. 57(2), 587-597 (2009)

18. Li, Y., Huang, X., Liu, D., et al.: Hybrid energy storage system and energy distribution strategy for four-wheel independent-drive electric vehicles. J. Clean. Prod. 220, 756-770 (2019)

19. Bai, Y., Li, J., He, H., et al.: Optimal design of a hybrid energy storage system in a plug-in hybrid electric vehicle for battery lifetime improvement. IEEE Access 8, 142148-142158 (2020)

20. Yang, B., Wang, J., Zhang, X., et al.: Applications of battery/ supercapacitor hybrid energy storage systems for electric vehicles using perturbation observer based robust control. J. Power Sources 448, 227444 (2020)

21. Li, L., Chen, H., Küçükay, F.: Systematic synthesis of dedicated hybrid transmission. Automot. Innov. 2(3), 231-239 (2019)

22. Tran, M.K., Akinsanya, M., Panchal, S., et al.: Design of a hybrid electric vehicle powertrain for performance optimization considering various powertrain components and configurations. Vehicles 3(1), 20-32 (2021) 
23. Zhao, H., Liu, C., Song, Z., et al.: Analytical modelling of a double-rotor multi-winding machine for hybrid aircraft propulsion. IEEE Trans. Transp. Electrif. 6(4), 1537-1550 (2020)

24. Zhang, F., Zhang, T., Yang, X., et al.: A high-performance supercapacitor-battery hybrid energy storage device based on graphene-enhanced electrode materials with ultrahigh energy density. Energy Environ. Sci. 6(5), 1623-1632 (2013)

25. Li, J., Xiong, R., Yang, Q., et al.: Design/test of a hybrid energy storage system for primary frequency control using a dynamic droop method in an isolated microgrid power system. Appl. Energy 201, 257-269 (2017)

26. Huang, Y., Wang, H., Khajepour, A., et al.: A review of power management strategies and component sizing methods for hybrid vehicles. Renew. Sustain. Energ. Rev. 96, 132-144 (2018)

27. Wieczorek, M., Lewandowski, M.: A mathematical representation of an energy management strategy for hybrid energy storage system in electric vehicle and real time optimization using a genetic algorithm. Appl. Energy 192, 222-233 (2017)

28. Yuan, J., Yang, L., Chen, Q.: Intelligent energy management strategy based on hierarchical approximate global optimization for plug-in fuel cell hybrid electric vehicles. Int. J. Hydrog. Energy 43(16), 8063-8078 (2018)
29. Eldeeb, H.H., Elsayed, A.T., Lashway, C.R., et al.: Hybrid energy storage sizing and power splitting optimization for plug-in electric vehicles. IEEE Trans. Ind. Appl. 55(3), 2252-2262 (2019)

30. Kelly, J.J., Leahy, P.G.: Sizing battery energy storage systems: using multi-objective optimization to overcome the investment scale problem of annual worth. IEEE Trans. Sustain. Energy 11(4), 2305-2314 (2019)

31. Ravey, A., Watrin, N., Blunier, B., et al.: Energy-source-sizing methodology for hybrid fuel cell vehicles based on statistical description of driving cycles. IEEE Trans. Veh. Technol. 60(9), 4164-4174 (2011)

32. Chen, G., Zhang, W.: Hierarchical coordinated control method for unmanned robot applied to automotive test. IEEE Trans. Ind. Electron. 63(2), 1039-1051 (2016)

33. Gee, T., Robinson, F., Dunn, R.: Analysis of battery lifetime extension in a small-scale wind-energy system using supercapacitors. IEEE Trans. Energy Convers. 28(1), 24-33 (2013)

34. Li, J., Yang, Q., Robinson, F., et al.: Design and test of a new droop control algorithm for a SMES/battery hybrid energy storage system. Energy 118, 1110-1122 (2017) 